Supplement of

\title{
Generating seamless global daily AMSR2 soil moisture (SGD-SM) long- term products for the years 2013-2019
}

\author{
Qiang Zhang et al. \\ Correspondence to: Qiangqiang Yuan (qqyuan@sgg.whu.edu.cn) and Liangpei Zhang (zlp62@whu.edu.cn)
}

The copyright of individual parts of the supplement might differ from the article licence. 
This document mainly demostrates the technical supplement of the network implementation. Detailed descriptions are depicted below:

1) 'The loss convergent model': The loss convergence model denotes that the loss of the proposed model gradually decreases, and finally maintains smooth in training procedure.

2) Reason for each number: ' $T$ ' stands for current daily date. ' $3 \times 3 \times 3$ ' refers to the kernel size of 3D convolutional cube filter. '11 layers' represents the depth of the proposed deep neural network. '90' is the feature map number in CNN. '0.1' denotes the balancing factor to adjust the local loss and global loss in Eq. (6). '128' stands for the batch size in deep learning model. '0.001' refers to the learning rate for the training procedure.

3) Relation between loss function and learning parameters: In deep learning theory, the loss function is the 'baton' of the whole network, which guides the network parameters learning through the error back-propagation between the predicted sample and the original sample. In terms of the learning parameters, they represent the weighted and bias parameters in all the layers.

4) How to understand 'epochs, Adam algorithms and the gradient descent strategy': One 'Epoch' represents that the network goes through all the training data. 'Adam algorithms' is a gradient descent method in back-propagation step, to optimize the whole network parameters. 'gradient descent' denotes the partial differentiation and then updates the variation for each network parameter, which obeys the chain rule in deep neural network.

5) 3D CNN filters: 3D CNN filters are employed to simultaneously capture both spatial and temporal soil moisture information in time-series products. For large gap areas, partial CNN is developed to exclude the invalid AMSR2 soil moisture information.

6) Layers: More layers in deep neural network can extract more intrinsic feature information for soil moisture products gap-filling. 
7) Feature maps: Feature maps get the description of the original soil moisture products from multiple angles, through different $3 \mathrm{D}$ CNN filters. The feature map of final layer is fixed as 1 , to export the reconstructing daily soil moisture result in date $T$.

For clearly understanding the parameters chosen in the proposed network such as 3D CNN filters, layer numbers, and feature maps, we have supplemented the sensitivity analysis of these parameters in discussion part. As listed in Table S1, Table S2, and Table S3, discussions for the 3D CNN filters, layer numbers, and feature maps are investigated in simulated missing regions validation, respectively. Accordingly, the optimal indexes are chosen as the setting value.

Table S1. Discussion for the 3D CNN filters in simulated missing regions validation

\begin{tabular}{cccc}
\hline \multirow{2}{*}{ Parameter } & \multicolumn{3}{c}{ Evaluation index } \\
\cline { 2 - 4 } & $\mathrm{R}$ & RMSE & MAE \\
\hline $3 \times 3 \times 3$ & $\mathbf{0 . 9 6 8}$ & $\mathbf{0 . 0 6 8}$ & $\mathbf{0 . 0 4 7}$ \\
\hline $5 \times 5 \times 5$ & 0.957 & 0.076 & 0.048 \\
\hline $7 \times 7 \times 7$ & 0.949 & 0.081 & 0.050 \\
\hline
\end{tabular}

Table S2. Discussion for the layer numbers in simulated missing regions validation

\begin{tabular}{cccc}
\hline \multirow{2}{*}{ Parameter } & \multicolumn{3}{c}{ Evaluation index } \\
\cline { 2 - 4 } & $\mathrm{R}$ & RMSE & MAE \\
\hline 10 & 0.962 & 0.072 & 0.048 \\
\hline 11 & $\mathbf{0 . 9 6 8}$ & $\mathbf{0 . 0 6 8}$ & $\mathbf{0 . 0 4 7}$ \\
\hline 12 & 0.966 & 0.070 & 0.049 \\
\hline
\end{tabular}

Table S3. Discussion for the feature maps in simulated missing regions validation

\begin{tabular}{cccc}
\hline \multirow{2}{*}{ Parameter } & \multicolumn{3}{c}{ Evaluation index } \\
\cline { 2 - 4 } & $\mathrm{R}$ & RMSE & MAE \\
\hline 60 & 0.963 & 0.071 & 0.048 \\
\hline 90 & $\mathbf{0 . 9 6 8}$ & $\mathbf{0 . 0 6 8}$ & $\mathbf{0 . 0 4 7}$ \\
\hline 120 & 0.967 & 0.069 & 0.047 \\
\hline
\end{tabular}

